\title{
29
}

\section{Towards a helioseismic calibration of the equation of state of the plasma in the solar convective envelope}

S. V. VORONTSOV ${ }^{1,2}$, V. A. BATURIN ${ }^{1,3}$, D. O. GOUGH ${ }^{4,1} \&$ W. DÄPPEN ${ }^{5}$

\footnotetext{
1 Astronomy Unit, Queen Mary and Westfield College, Mile End Road, London E1 $\{N S, C K$

2 Institute of Physics of the Earth, B.Gruzinskaya 10, Moscou 129810, Russia (permanent address)

${ }^{3}$ Sternberg Astronomical Institute, Cniversitetsky Prospect 19, Moscou 119899, Russia (permanent address)

4 Institute of Astronomy, and Department of Applied Mathematics and. Theoretical Phgsics, Madingley Road, Cambridge CBY OHA, UK

5 Department of Physics and Astronomy, University of Southern California, Los Angeles, CA 90089-1942, L'SA
}

\begin{abstract}
We report the results of a nonlinear inversion of solar oscillation data that enable us to detect nonideal Coulomb interactions between particles, including pressure ionization, in the solar convection zone.
\end{abstract}

\subsection{Introduction}

Precise measurements of solar oscillation frequencies provide data for accurate inversions for the sound speed in the solar interior. Except in the very outer layers, the stratification of the convection zone is almost adiabatic. There, the sound-speed profile is governed principally by the specific entropy, the chemical composition and the equation of state, being essentially independent of the uncertainties in the radiative opacities. The inversions thus reveal, via tiny effects on the adiabatic compressibility of the solar plasma, physical processes that influence slightly the equation of state. We have carried out a nonlinear inversion based on a recent accurate asymptotic description of intermediate- and high-degree solar p modes (Brodsky \&. Vorontsov 1993; Gough \& Vorontsov 1993), using the observational data of Libbrecht, Woodard \& Kaufman (1990). 


\subsection{The equations of state (EOS) used in the analysis}

In the reference models, we use the following equations of state. We are mostly brief, with the exception of the pressure-ionization model used in the helioseismic calibration.

- Saha EOS: a free-energy-minimization type realization for a mixture of reacting ideal gases, with ground-state-only partition functions of the bound species. Note that by assuming only ground states we are using the term 'Saha' in a rather restricted sense.

- Debye-Hückel (DH) EOS: the Saha EOS together with a consistent free-energy term for the Coulomb interaction in the Debye-Hückel approximation.

- PI(S) EOS: a simple model to reproduce the essential thermodynamic features of pressure ionization of hydrogen, neutral helium and singly-ionized helium (we neglect the effect of the pressure ionization of the elements beyond hydrogen and helium because of their small abundance). This model, inspired by the confined-atom approach (e.g. Däppen, 1980), includes only ground states of particles, and describes the main effect of pressure ionization as a smooth reduction of the effective partition function as density increases:

$$
Z_{\mathrm{eff}}^{i}=Z_{0}^{i} \exp -\left(\frac{\lambda R_{i}}{R}\right)^{3}
$$

In this expression $R$ denotes the mean distance between the centres of nearest neighbours (of all species, charged and neutral), $R_{i}$ is the radius of species $i$ (we adopt $R_{\mathrm{H}}=R_{\mathrm{He}}=1$, and $R_{\mathrm{He}^{+}}=0.5$ ), and $\lambda$ is a parameter that we adjust to solar data (in the following PI(S) EOS means such an adjusted equation of state with $\lambda=1.667$ ). Expression (1) for $Z_{\mathrm{eff}}^{i}$ is then incorporated into DH EOS in a consistent way.

- MHD EOS: the free-energy-minimization realization of the Hummer \& Mihalas (1988) occupation-probability formalism (Mihalas, Däppen \& Hummer, 1988; Däppen et al., 1988). A large number of species are interacting with each other; the destruction of extended species is taken into account by both volume and charge effects. The MHD equation of state includes the $\mathrm{DH}$ terms too.

\subsection{Results}

We show the results of our inversions for various reference models based on the different equations of state described above, and we compare each model with observations. We plot the sound-speed gradient [in the dimen- 


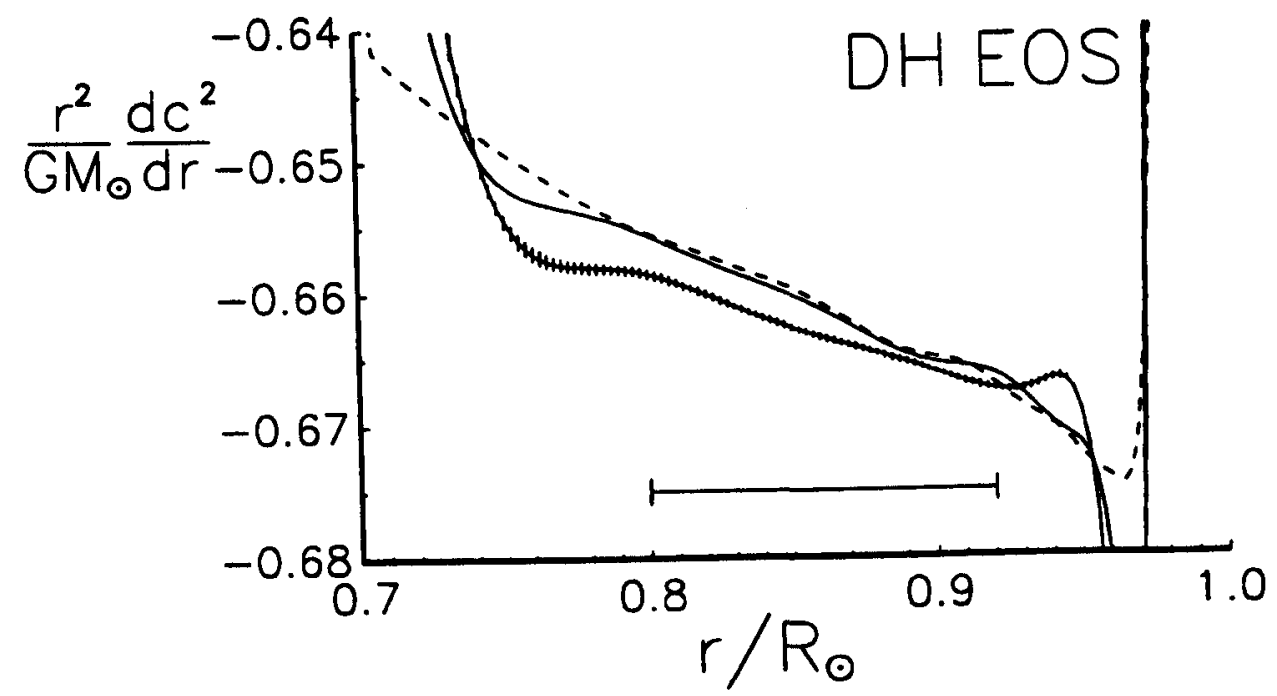

Fig. 29.1 Sound-speed gradient in the solar convection zone. Dashed line: reference model computed with the DH EOS. Solid line: inversion of artificial data computed with reference model. Solid line with error bars: inversion of observational frequencies. The horizontal bar indicates the region where the inversion is most accurate and reliable. Note the significant. discrepancy between theory and observation.

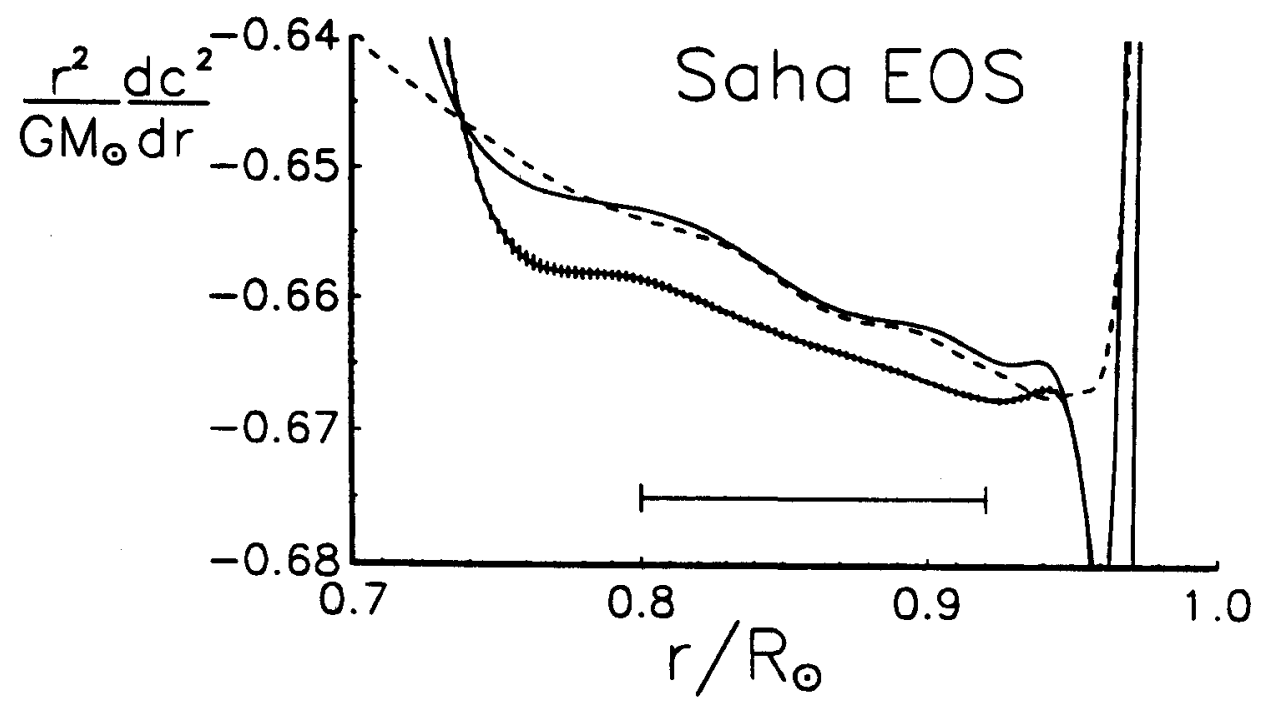

Fig. 29.2 Same as Fig. 1, but with an equation of state that has no electrostatic interaction term (Saha EOS). The discrepancy between theory and observation becomes larger.

sionless form used in studies of the helium abundance (Gough, 1984; see also the recent review by Kosovichev et al., 1992). The sound-speed gradient obtained for the Sun is compared with that of our reference models: DH-EOS (Fig. 1), Saha EOS (Fig. 2), PI(S) EOS (Fig. 3), DH EOS for two 


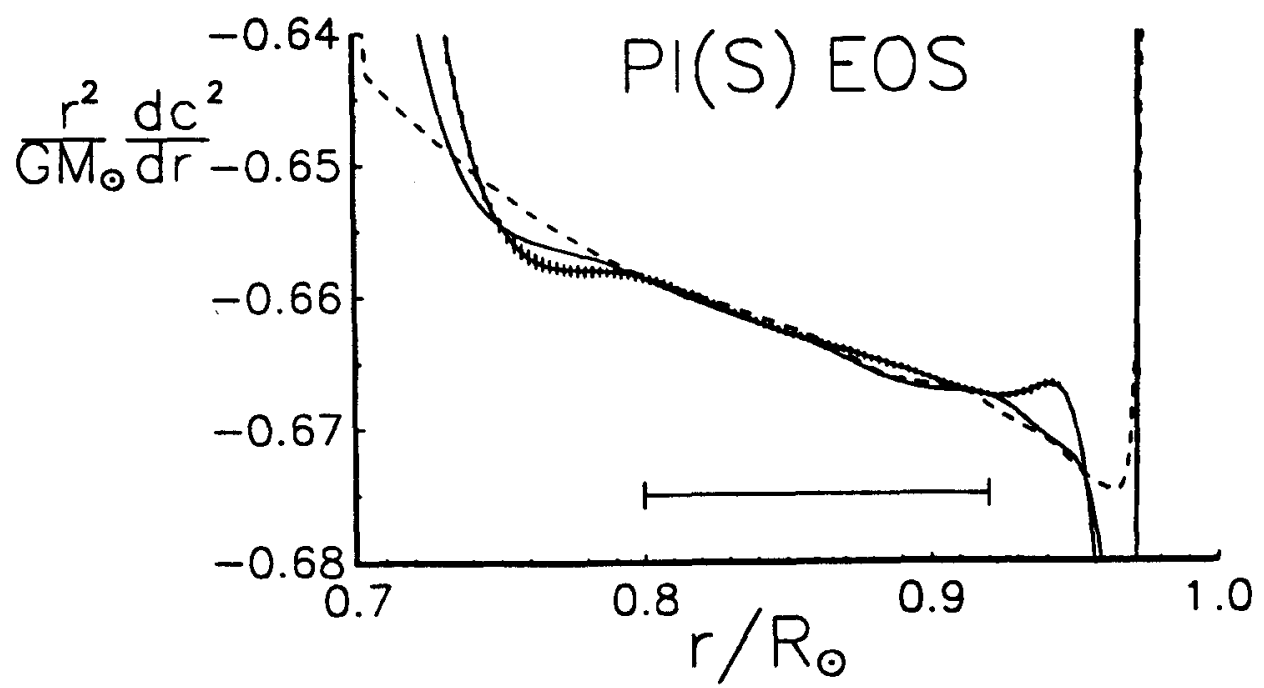

Fig. 29.3 The discrepancy between the model and the inverted solar data can be removed by taking appropriate account of pressure ionization [PI(S)].

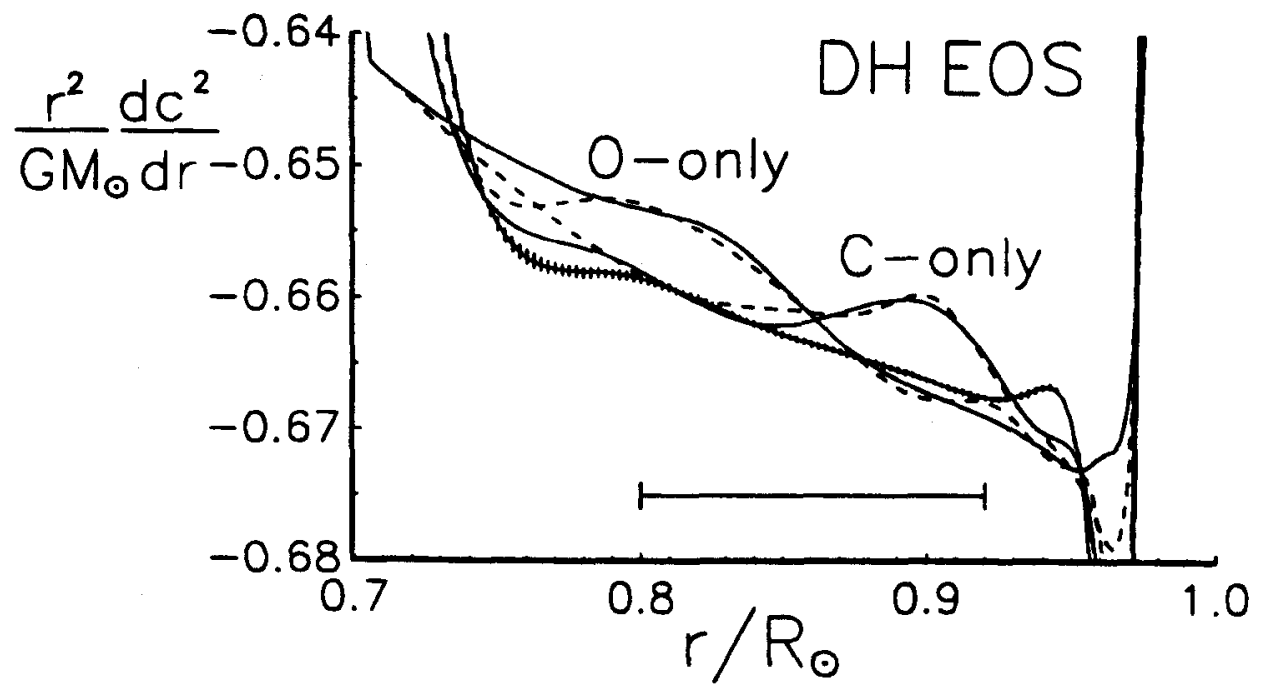

Fig. 29.4 Same as Fig. 1, but with two models in which all the heavy elements have been replaced by either oxygen ( $\mathrm{O}$-only) or carbon ( $\mathrm{C}$-only).

selected choices of heavy-element abundance ( $\mathrm{C}$ only and $\mathrm{O}$ only) (Fig. 4), and in Fig. 5 the MHD equation of state (also for two different choices of heavy-element abundance).

\subsection{Conclusion}

The accuracy of the inversion that is achieved with the observational data currently available permits us to detect the influence of Coulomb interac- 


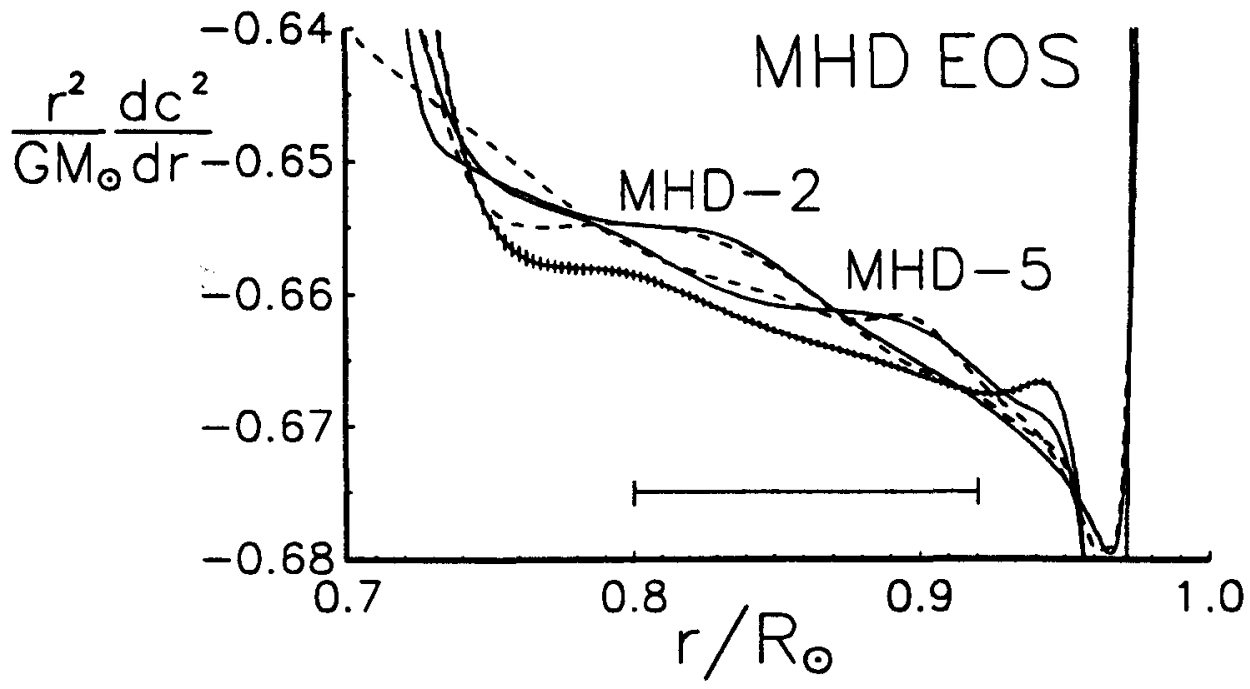

Fig. 29.5 Same as Fig. 1, but with two models computed with the MihalasHummer-Däppen (MHD) equation of state. MHD-5 refers to a more complete set in which (besides $\mathrm{H}$ and $\mathrm{He}$ ) $\mathrm{C}, \mathrm{N}, \mathrm{O}$, and $\mathrm{Fe}$ are treated in all details, and all species interact with each other. MHD-2 stands for a simpler chemical mixture, with oxygen alone replacing all heavy elements.

tions between particles. At the same time we have established a clear diagnostic potential to probe the mixture of heavy elements in the convection zone. We are thus putting constraints on the physical foundation and the chemical composition of the equation of state. In this way we can assess the quality of the different versions of the equation of state that are currently used for modelling the solar interior.

\section{References}

Brodsky, M. \& Vorontsov, S.V., Astrophys. J., 409, 455 (1993)

Däppen, Astron. Astrophys., 91, 212 (1980)

Däppen, W., Mihalas, D., Hummer, D.G. \& Mihalas, B.W., Astrophys. J., 332, $261(1988)$

Gough, D.O., Mem. Soc. Astron. Ital., 55, 13 (1984)

Gough, D.O. \& Vorontsov, S.V., Mon. Not. R. astr. Soc., (1993) submitted

Hummer, D.G. \& Mihalas, D., Astrophys. J., 331, 794 (1988)

Kosovichev, A.G., Christensen-Dalsgaard, J., Däppen, W., Dziembowski, W.A., Gough, D.O.,\& Thompson, M.J., Mon. Not. R. astr. Soc., 259, 536 (1992)

Libbrecht, K.G., Woodard, M.F. \& Kaufman, J.M., Astrophys. J. Suppl., 74, 1129 (1990)

Mihalas, D., Däppen W. \&. Hummer, D.G., Astrophys. J., 331, 815 (1988) 